\title{
Error Performance Analysis of Detect-and-Forward Superposed Cooperation
}

\author{
Ang Yang, Zesong Fei, Meng Zheng, Jingming Kuang \\ School of Information Science and technology \\ Beijing Institute of Technology \\ Beijing, China \\ Email: \{cool_yang, feizesong, ZMRA\}@bit.edu.cn
}

\begin{abstract}
This paper proposes a closed form of error performance of an un-coded detect-and-forward superposed relay in term of equivalent SNR. The theoretical results show excellent correspondence with computer simulations. It has been proved that power allocation has great effect on the performance of superposed cooperation. The analysis makes it possible to optimize the power allocation between the local and relay transmissions numerically.
\end{abstract}

Index Terms-superposed cooperation, error probability, equivalent SNR, power allocation

\section{INTRODUCTION}

Cooperative communication has attracted lots of attentions because of the gain brought by the spatial diversity. In traditional decode-and-forward(DF) cooperation scheme as illustrated in Figure 1, Node B first decodes the packet from Node A, and, if successful, re-encodes and retransmits it, possibly using a different channel code. If Node A decodes unsuccessfully, it transmits its own data. One clear disadvantage of DF is the about $50 \%$ loss of spectral efficiency caused by the retransmission at the relay node. To improve the spectrum efficiency, a new cooperation scheme named superposed cooperation is proposed in [1]. Some research has been done on superposed cooperation [2] [3] [4] [5] [6] [7].

As shown in Figure 1, during each time slot, Node A and Node B transmit in turn to deliver their packets to a common destination D. To exploit spatial diversity and thereby enhance reliability on fading channels, each source node transmits both its own "local" packet as well as a "relay" packet that originated from its partner. Figure 2 shows the constellation of superposed BPSK signal which has the similar shape of 4PAM modulation. The symbol $\delta^{2}$ is the ratio of the power allocation for the partner. It has been shown in [1] that compared with the classical cooperation in the same rates, superposed cooperation can provide better performance.

As shown in Figure 2, the power allocation $\delta^{2}$ has great effect on the shape of the constellation so that it obviously influences the performance of the scheme. Some research has

Zesong Fei(corresponding aurthor, phone: +86-10-68915838, email: feizesong@bit.edu.cn), Ang Yang, Meng Zheng and Jingming Kuang are with the School of Information and Electronics, Beijing Institute of Technology, Beijing 100081, China. The work of this paper was supported in part by the Sino-Swedish IMT-Advanced and Beyond Cooperative Program under grant No.2008DFA11780 and by Ericsson.

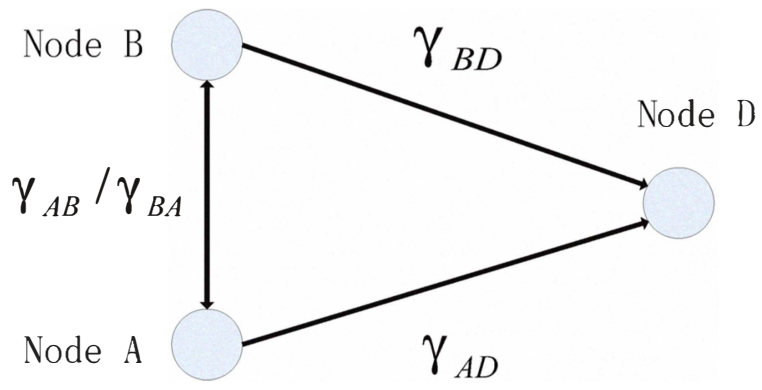

Fig. 1. System model

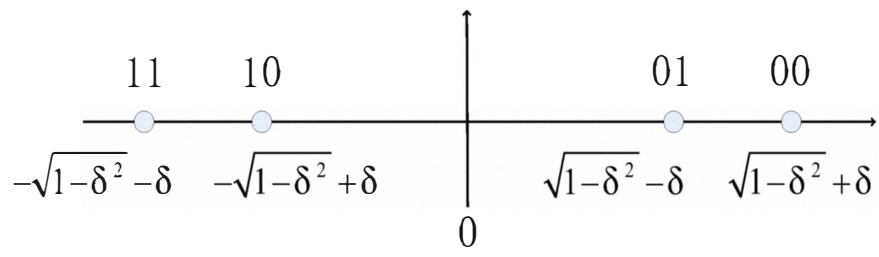

Fig. 2. Constellation of superposed cooperation

been done to find out the best the power allocation in theory [2] [4]. Reference [2] proposes a function to characterize how equivalent SNR depends on the SNRs of the desired bit and the superimposed bit, based on log likelihood ratio (LLR) with convolutional code. An analytical performance bound for a coded cooperative diversity signal superposition system is developed in [2].

This paper focuses on un-coded detect-and-forward superposed relay and proposes a closed form of error performance of the scheme, which is based on equivalent SNR. The presented closed expressions of equivalent SNR are used to search the power allocation ratio that is used to transmit the information of itself and of the partner. The analytical results facilitate numerical optimization of the power allocation without adopting computationally intensive simulations.

\section{ERROR PERFORMANCE ANALYSIS}

As shown in Figure 1, the system consists of 3 nodes. Node A and Node B are transmitters in the same situation, and Node $\mathrm{D}$ is destination node which receives the signal from the other two. The $E_{s} / N_{0}$ of the link is denoted by $\gamma$. 


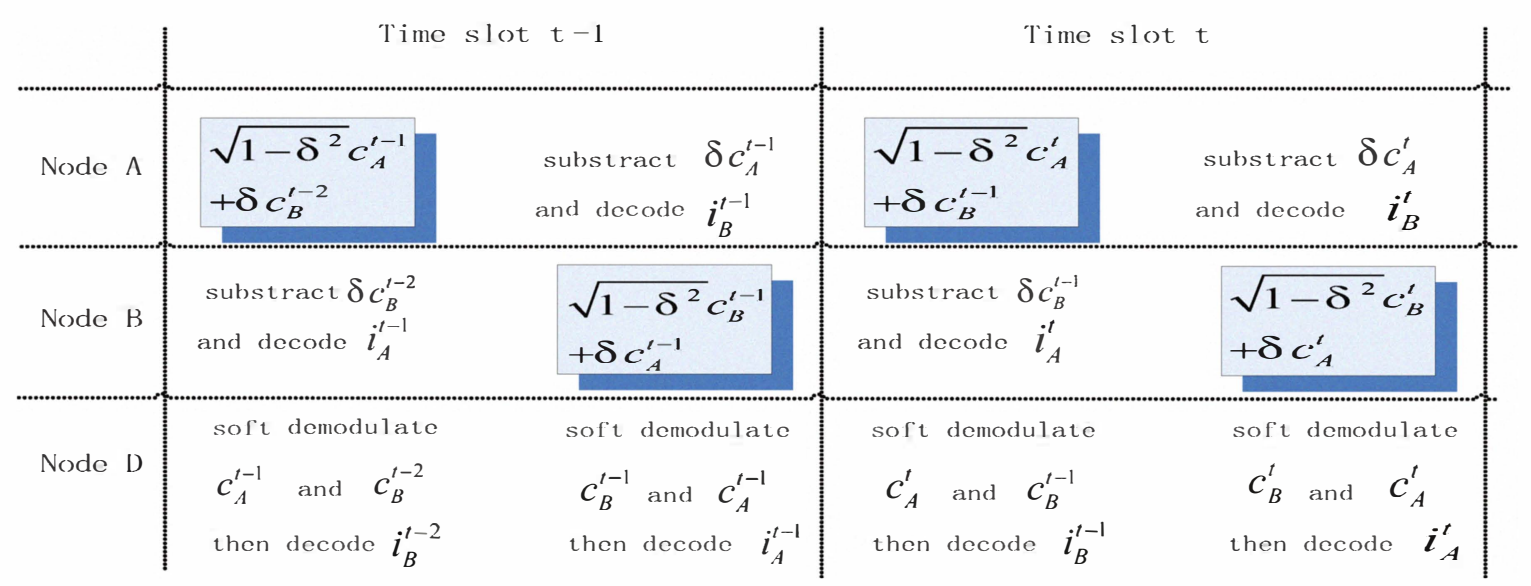

Fig. 3. The operations in superposition modulation system.Here the boxes depict the transmitted signals, $c$ stands for BPSK modulated symbols, $i$ means the information bits, and $\delta$ is the power allocation.

For each of the transmitter, the transmission can mainly be divided into two phases. In the first phase, the node listens to the partner and tries to decode the information. And in the second phase, if decodes successfully, the node re-encodes the partner's information, superposes it with signal generated by itself, and broadcasts the signal to both the partner and the destination. Else only its own signal is broadcasted. When Node D receives the superposed signal, Node D demodulates it and combines the soft values with those got from last frame.

To be mentioned, in the situation that the node receives superposed signal from the partner, it can divide its own part which was transmitted in the last frame, and thus transforms the signal to BPSK modulated, with the $E_{s} / N_{0}$ equals to $\left(1-\delta^{2}\right) \gamma$. For simplify, we assume the situation that the channel between Node A and Node B is error free.

Figure 3 depicts the operations at Node A, Node B and Node D during time slots $\mathrm{t}-1$ and $\mathrm{t}$ for the scheme under consideration. All channels are subject to independent block Rayleigh fading. During time slot $\mathrm{t}-1$, a superposed signal that Node D receives from Node A can be described as

$$
y_{D 1}=\sqrt{f_{A D}}\left(\sqrt{1-\delta^{2}}(-1)^{s_{1}}+\delta(-1)^{i_{1}}\right)+n
$$

where $y_{D_{1}}$ represents the superposed signals from Node $\mathrm{A}$, $f_{A D}$ is fading coefficient, $\delta$ is the power allocation, $s_{1}$ stands for the bit of Node A, $i_{1}$ means the bit of Node B and $n$ is zero mean additive white Gaussian noise. In this case, $s_{1}$ is the "local" bit and $i_{1}$ is the "relay" bit.

Without loss of generality, we assume that the value of $s_{1}$ is 0 . When the value of $i_{1}$ is 0 , the probability that Node $\mathrm{D}$ obtains the wrong bit of $s_{1}$ can be expressed as

$$
\begin{aligned}
& P_{e}\left(s_{1} \mid s_{1}=0, i_{1}=0\right) \\
& =\int_{0}^{\infty} Q\left(\sqrt{2 f_{A D}\left(\sqrt{1-\delta^{2}}+\delta\right)^{2} \gamma_{A D}}\right) e^{-f_{A D} d f_{A D}} \\
& =\frac{1}{2}\left[1-\sqrt{\frac{\left(\sqrt{1-\delta^{2}}+\delta\right)^{2} \gamma_{A D}}{1+\left(\sqrt{1-\delta^{2}}+\delta\right)^{2} \gamma_{A D}}}\right]
\end{aligned}
$$

The modulation seems to be BPSK for Node A's information with a known binary interference. So Node A's equivalent SNR for this case takes the form

$$
\bar{\gamma}_{A_{1,0}}=\left(\sqrt{1-\delta^{2}}+\delta\right)^{2} \gamma_{A D}
$$

When the value of $i_{1}$ is 1 , the probability that Node D acquires the wrong bit of $s_{1}$ is

$$
\begin{aligned}
& P_{e}\left(s_{1} \mid s_{1}=0, i_{1}=1\right) \\
& =\int_{0}^{\infty} Q\left(\sqrt{2 f_{A D}\left(\sqrt{1-\delta^{2}}-\delta\right)^{2} \gamma_{A D}}\right) e^{-f_{A D}} d f_{A D} \\
& =\frac{1}{2}\left[1-\sqrt{\frac{\left(\sqrt{1-\delta^{2}}-\delta\right)^{2} \gamma_{A D}}{1+\left(\sqrt{1-\delta^{2}}-\delta\right)^{2} \gamma_{A D}}}\right]
\end{aligned}
$$

Node A's equivalent SNR for this case takes the form

$$
\bar{\gamma}_{A_{1,1}}=\left(\sqrt{1-\delta^{2}}-\delta\right)^{2} \gamma_{A D}
$$

During time slot $\mathrm{t}$, a received superposed signal at Node D which transmitted from Node B can be described as

$$
y_{D_{2}}=\sqrt{f_{B D}}\left(\sqrt{1-\delta^{2}}(-1)^{i_{2}}+\delta(-1)^{s_{1}}\right)+n
$$

However, in this case, $y_{D_{2}}$ denotes the superposed signals from Node $\mathbf{B}, f_{B D}$ is fading coefficient, $i_{2}$ is the "local" bit and $s_{1}$ is the "relay" bit.

When the value of $i_{2}$ is 0 , the probability that $\mathrm{D}$ obtains the wrong bit of $s_{1}$ can be expressed as (7).

Node A's equivalent SNR for this case takes the form

$$
\bar{\gamma}_{A_{2,0}} \approx \frac{\gamma_{B D}}{\frac{1}{\delta^{2}}-\frac{1}{\left(\sqrt{1-\delta^{2}}+\delta\right)^{2}}+\frac{1}{\left(2 \sqrt{1-\delta^{2}}+\delta\right)^{2}}}
$$

When the value of $i_{2}$ is 1 , Node A's equivalent SNR for this condition can be described as

$$
\bar{\gamma}_{A_{2,1}} \approx \frac{\gamma_{B D}}{\frac{1}{\delta^{2}}+\frac{1}{\left(\sqrt{1-\delta^{2}}-\delta\right)^{2}}-\frac{1}{\left(2 \sqrt{1-\delta^{2}}-\delta\right)^{2}}}
$$




$$
\begin{aligned}
& P_{e}\left(s_{1} \mid s_{1}=0, i_{2}=0\right) \\
& =\int_{0}^{\infty} Q\left(\sqrt{2 f_{B D} \delta^{2} \gamma_{B D}}\right) e^{-f_{B D}} d f_{B D}-\int_{0}^{\infty} Q\left(\sqrt{2 f_{B D}\left(\sqrt{1-\delta^{2}}+\delta\right)^{2} \gamma_{B D}}\right) e^{-f_{B D}} d f_{B D} \\
& +\int_{0}^{\infty} Q\left(\sqrt{\left.2 f_{B D}\left(2 \sqrt{1-\delta^{2}}+\delta\right)^{2} \gamma_{B D}\right) e^{-f_{B D}} d f_{B D}}\right. \\
& =\frac{1}{2}\left[1-\sqrt{\frac{\delta^{2} \gamma_{B D}}{1+\delta^{2} \gamma_{B D}}}\right]-\frac{1}{2}\left[1-\sqrt{\frac{\left(\sqrt{1-\delta^{2}}+\delta\right)^{2} \gamma_{B D}}{1+\left(\sqrt{1-\delta^{2}}+\delta\right)^{2} \gamma_{B D}}}\right]+\frac{1}{2}\left[1-\sqrt{\frac{\left(2 \sqrt{1-\delta^{2}}+\delta\right)^{2} \gamma_{B D}}{1+\left(2 \sqrt{1-\delta^{2}}+\delta\right)^{2} \gamma_{B D}}}\right] \\
& \approx \frac{1}{4 \gamma_{B D}}\left(\frac{1}{\delta^{2}}-\frac{1}{\left(\sqrt{1-\delta^{2}}+\delta\right)^{2}}+\frac{1}{\left(2 \sqrt{1-\delta^{2}}+\delta\right)^{2}}\right)
\end{aligned}
$$

Using Bayesian formula, the probability the Node D gets the wrong bit of $s_{1}$ can be depicted as

$$
\begin{aligned}
& P_{e}\left(s_{1}\right) \\
& =P_{e}\left(s_{1} \mid s_{1}=0\right) P\left(s_{1}=0\right)+P_{e}\left(s_{1} \mid s_{1}=1\right) P\left(s_{1}=1\right) \\
& =P_{e}\left(s_{1} \mid s_{1}=0\right) \\
& =\sum_{j=0}^{1} \sum_{k=0}^{1} P_{e}\left(s_{1} \mid s_{1}=0, i_{1}=j, i_{2}=k\right) P\left(i_{1}=j\right) P\left(i_{2}=k\right)
\end{aligned}
$$

where the assumption that the source nodes transmit 0 and 1 with the same probability is used.

Node $\mathrm{D}$ collects these copies with a maximum ratio combining (MRC). Instantaneous SNR of Node A can be calculated as

$$
\gamma_{A}=f_{A D} \bar{\gamma}_{A_{1, j}}+f_{B D} \bar{\gamma}_{A_{2, k}}
$$

where $j$ and $k$ can be 0 or 1 independently.

Equation (12) is the closed expression of evaluating Node A's bit error probability. In(12), $\bar{\gamma}_{A_{1, j}}$ and $\bar{\gamma}_{A_{2, k}}$ can be generated from $(3)(5)(8)(9)$.

\section{NUMERICAL EXAMPLE}

We present a numerical result to show the performance of the error probability analysis. All channels are subject to independent block Rayleigh fading. As mentioned before, we consider the un-coded detect-and-forward superposed cooperation scheme, assuming that the channel between Node $A$ and Node B is error free.

In Figure 4 , the numerical bit error probability curves are compared with simulation, with the power allocation $\delta^{2}=0.05$ , $\delta^{2}=0.15$ and $\delta^{2}=0.35$. In these cases, the analytical results seem to track the simulation results well. For example, the numerical curve is only less than $0.1 \mathrm{~dB}$ from the simulation curve when the average SNR is $15 \mathrm{~dB}$. When calculating the equivalent SNR of the "relay" bit, an approximation is used to simplify the expression form. It seems that the approximation is the main factor that causes the disparity.

It is shown from Figure 4 that the power allocation $\delta^{2}$ plays an important role in the BER. So $\delta^{2}$ should be chosen to

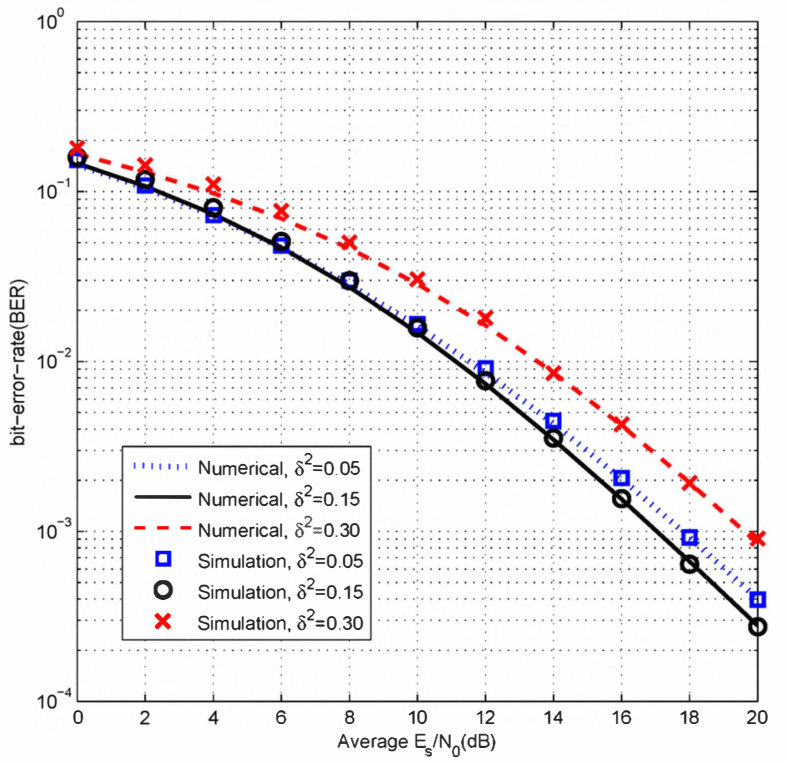

Fig. 4. Bit error rate for Rayleigh fading channels versus the average SNRs of channel A-D and B-D (both SNRs are the same).

minimize the BER. The effects of $\delta^{2}$ on BER performance are shown in Figure 5 for average SNRs of $10 \mathrm{~dB}$ and $20 \mathrm{~dB}$. The analytical results are almost equal to simulation results. The optimal power allocation $\delta^{2}$ is in the range 0.1 to 0.15 , which is identical with the conclusions of [1] and [2]. Comparing the $10 \mathrm{~dB}$ case to the $20 \mathrm{~dB}$ case, it is apparent that the power allocation has a more significant impact at higher SNRs.

In Figure 6, we plot the optimal power allocation coefficient $\delta^{2}$ as a function of the average SNR. The optimality is in the sense of minimizing the numerical bit error probability and the optimal $\delta^{2}$ is found by searching over the interval $(0,0.45)$. The optimal $\delta^{2}$ is less than 0.1 for low SNR. It can be comprehend that the information sent by the partner don't make any sense to improve the system performance but may be a interference. So the superposed cooperation does't work very well for low SNR. In the condition of high 


$$
\begin{aligned}
& P_{e}\left(s_{1}\right)=\frac{1}{4} \sum_{j=0}^{1} \sum_{k=0}^{1} \int_{0}^{\infty} \int_{0}^{\infty} Q\left(\sqrt{2\left(f_{A D} \bar{\gamma}_{A 1, j}+f_{B D} \bar{\gamma}_{A 2, k}\right)}\right) e^{-f_{A D} e^{-f_{B D}} d f_{A D} d f_{B D}} \\
& =\frac{1}{4} \sum_{j=0}^{1} \sum_{k=0}^{1} \int_{0}^{\infty} \int_{0}^{\infty} \frac{1}{\pi} \int_{0}^{\frac{\pi}{2}} e^{-\frac{f_{A D} \bar{\gamma}_{A 1, j}+f_{B D} \bar{\gamma}_{A 2, k}}{\sin ^{2} \phi}} e^{-f_{A D} e^{-f_{B D}} d f_{A D} d f_{B D}} \\
& =\frac{1}{4} \sum_{j=0}^{1} \sum_{k=0}^{1} \frac{1}{\pi} \int_{0}^{\frac{\pi}{2}} \frac{\sin ^{2} \phi}{\sin ^{2} \phi+\bar{\gamma}_{A 1, j}} \frac{\sin ^{2} \phi}{\sin ^{2} \phi+\bar{\gamma}_{A 2, k}} d \phi \\
& =\frac{1}{4} \sum_{j=0}^{1} \sum_{k=0}^{1}\left(\frac{1}{2}+\frac{1}{2} \frac{\bar{\gamma}_{A 1, j}}{\bar{\gamma}_{A 2, k}-\bar{\gamma}_{A 1, j}} \sqrt{\frac{\bar{\gamma}_{A 1, j}}{1+\bar{\gamma}_{A 1, j}}}-\frac{1}{2} \frac{\bar{\gamma}_{A 2, k}}{\bar{\gamma}_{A 2, k}-\bar{\gamma}_{A 1, j}} \sqrt{\frac{\bar{\gamma}_{A 2, k}}{1+\bar{\gamma}_{A 2, k}}}\right)
\end{aligned}
$$

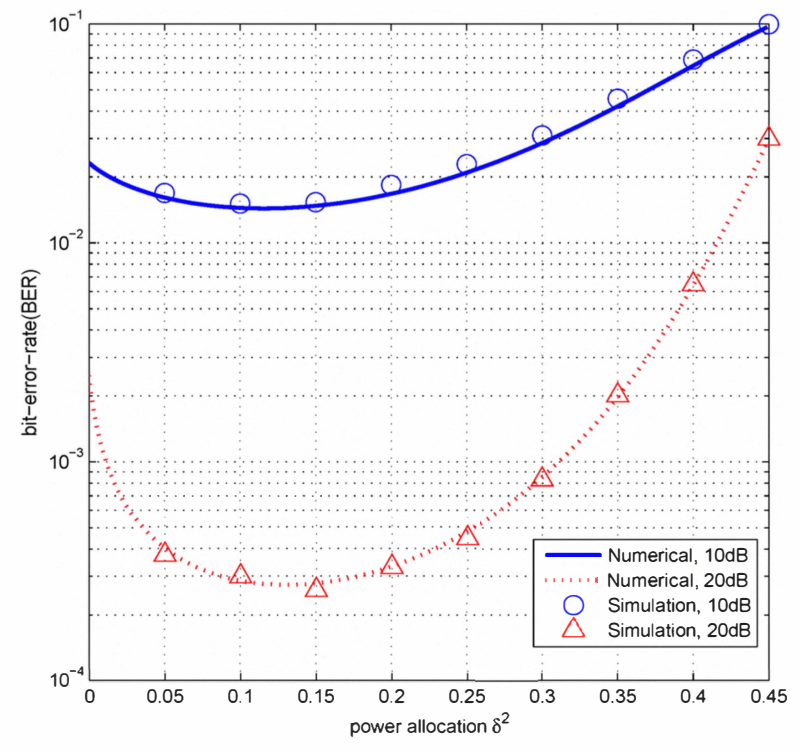

Fig. 5. Bit error rate as functions of the power allocation $\delta^{2}$ at an average $E_{s} / N_{0}$ of $10 \mathrm{~dB}$ and $20 \mathrm{~dB}$,when the average SNRs of channel A-D and B-D are the same.

SNR, the superposed cooperation becomes more available and can improve the system performance. Results may differ for different channel models and different code generators.

\section{CONCLUSION}

Based on equivalent SNR expression, we have presented an error performance analysis of an un-coded detect-and-forward superposed cooperation scheme. Minimizing the bound shows the dependence of the optimum superposition factor on the operating SNR. The closed expressions of equivalent SNR and bit error probability are derived in this paper. The analytical results match to the computer simulations perfectly. This work can be extended to predict the performance and optimize the design of the general cooperation scheme, not only for superposed cooperation.

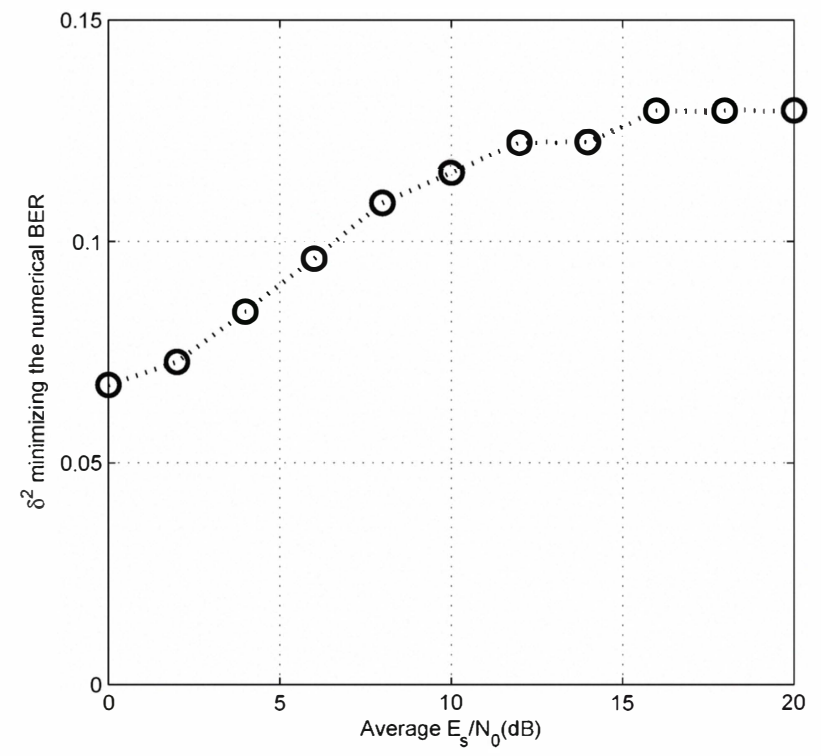

Fig. 6. Values of $\delta^{2}$ that minimize the numerical BER after the superposed cooperation versus the average SNRs of channel A-D and B-D (both SNRs are the same).

\section{REFERENCES}

[1] E. G. Larsson and B. R. Vojcic, "Cooperative transmit diversity based on superposition modulation," IEEE Communications Letters, vol. 9, pp. 778-780, Seqt 2005.

[2] J. K. X. Lei, T. E. Fuja and D. J. Costello, "Error performance analysis of signal superposition coded cooperative diversity," IEEE Trans. Commun., vol. 57, pp. 3123-3131, Oct 2009.

[3] K. Ishii, "Superposition modulated cooperative diversity for half-duplex scenario," J. Commun., vol. 2, pp. 20-27, Dec 2007.

[4] I. Krikidis, "Analysis and optimization issues for superposition modulation in cooperative networks," IEEE Trans. Veh. Technol, vol. 58, pp. 4837-4847, Nov 2009.

[5] X. Y. L. Ping, J. Tong and Q. Guo, "Superposition coded modulation and iterative linear mmse detection," IEEE J. Select. Areas Commun., vol. 27, pp. 995-1004, Aug 2009.

[6] T. Yang and J. Yuan, "Performance of iterative decoding for superposition modulation-based cooperative transmission," IEEE Trans. Wireless Commun., vol. 9, pp. 51-59, Jan 2010.

[7] T. R. Z. Ding and C. Cowan, "On the performance of superposition cooperative diversity in wireless networks," in Proc. IEEE Int. Symp. Inf. Theory, Seattle, WA, Jul. 2006. 